\title{
THE DISTRIBUTION OF COMMISSURAL FIBRES IN THE CORPUS CALLOSUM IN THE MACAQUE MONKEY
}

\author{
BY

\section{S. SUNDERLAND} \\ From the Department of Human Anatomy, Oxford
}

(RECEIVED 22ND JULY, 1939)

DURING an investigation to determine the projection of the cerebral cortex on to the pons and cerebellum by tracing fibres undergoing Marchi degeneration, a series of cortical lesions was made in macaque monkeys. In the preparation of this material for histological examination, the corpus callosum was sectioned to see which of the cortical areas studied gave origin to commissural fibres and to determine the relative position of these in the corpus callosum. Though the presence of callosal fibres is often recorded in contributions dealing with experimental studies on cortical projectional pathways, their position in the corpus callosum is not often referred to.

The observations are based on six experiments. The lesions were localized, so as to involve, as far as possible, not more than two cyto-architectural areas. They were produced by the method of devascularization, already described in a previous paper, which is based on the principle that, if the superficial pial vessels to a cortical area are obliterated, the cortex undergoes complete necrosis, while the changes in the subjacent white matter are, in general, restricted to that portion of it which is occupied predominantly by fibres arising in the involved cortex (Sunderland, 1938). In this method of devascularization, subjacent fibre pathways are thus likely to be preserved intact, and this is particularly important since it is essential that any fibre tracts of a possible commissural nature from adjacent cortical areas should be avoided.

The animals were anæsthetized by means of the intra-peritoneal injection of sodium ethyl barbiturate (nembutal), using 0.7 c.c. of a 5 per cent. solution per kilogram of body weight. In each case the left cerebral hemisphere was exposed by reflecting a rectangular osteo-plastic flap, and the lesion produced as described. After all bleeding had been carefully controlled the wound was closed. There were no post-operative complications. The animals were killed 17 days later by ether anæsthesia. The vascular system was perfused with 10 per cent. formalin after clearing it with repeated washings with normal saline by way of the aorta. The brain was removed 1 hour later, washed in 
running water, and the corpus callosum then exposed and removed. The block of tissue was mordanted in potassium bichromate and then passed through potassium bichromate containing various quantities of osmic acid, cleared in spirit, and finally embedded in celloidin. Sections were cut at $15 \mu$. The cortical lesions were carefully examined, measured, and drawn.

\section{Experimental results}

Experiment 1.-Monkey 45A. Operation 5th May, 1938. Killed 23rd May, 1938.

The lesion was situated in the prefrontal region involving most of area 8 , but also encroaching on areas 9 and 10. It measured $17 \mathrm{~mm}$. coronally and $15 \mathrm{~mm}$. sagittally. The anterior margin was $12 \mathrm{~mm}$. from the frontal pole,

$a$
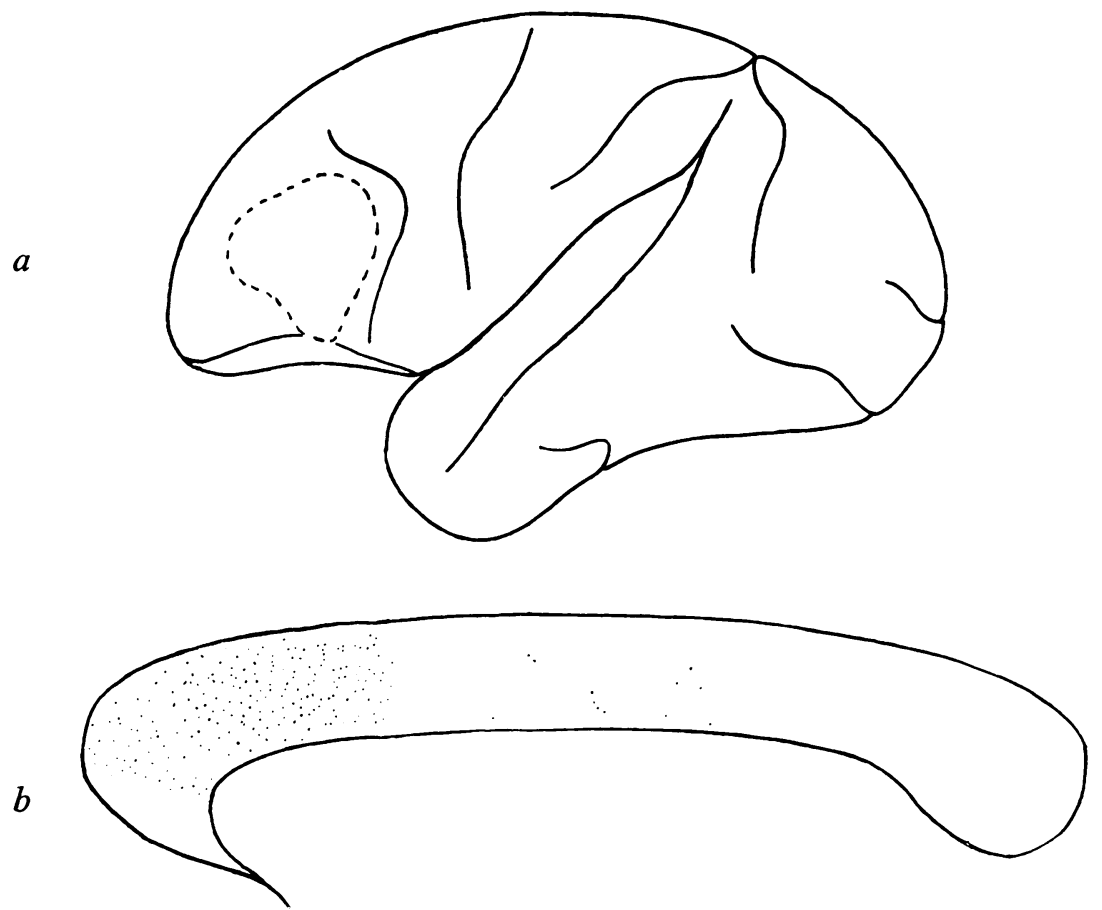

Fig. 1.-The lesion in experiment 1 and the distribution of the degenerated fibres in the corpus callosum.

while the posterior margin traced a parallel course $3 \mathrm{~mm}$. anterior to the sulcus arcuatus. Inferiorly it just reached the lower margin of the brain (Fig. 1, a).

In the sections of the corpus callosum fine Marchi granules were distributed evenly over the genu and the anterior third of the body, being densely arranged in the posterior part of this area. A few isolated granules were also seen elsewhere in the body, chiefly along its inferior aspect, but these were widely scattered and of doubtful significance. There was no Marchi deposit in the anterior commissure (Fig. 1, b). 
Experiment 2.-Monkey 46A. Operation 29th June, 1938. Killed 16th July, 1938.

The lesion was situated at the lower end of the upper limb of the sulcus lunatus and involved parts of areas 17, 18, and 19. The end of the sulcus was included in the lesion, the latter measuring $18 \mathrm{~mm}$. anteroposteriorly and $14 \mathrm{~mm}$. in a vertical direction. Anteriorly the margin reached almost to the temporal sulcus, while it was $24 \mathrm{~mm}$. from the occipital pole posteriorly and $7 \mathrm{~mm}$. from the lower border of the brain inferiorly (Fig. 2, $a$ ).

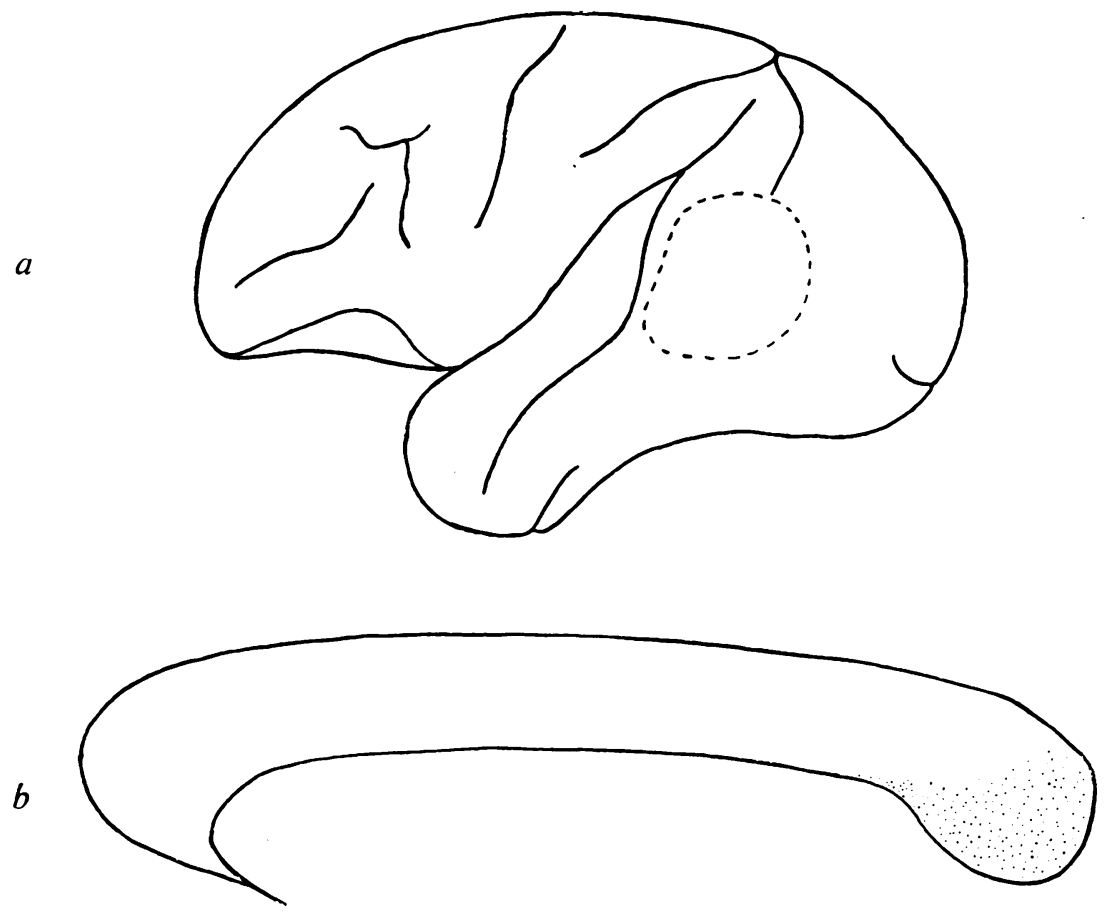

Fig. 2.-The lesion in experiment 2 and the distribution of the degenerated fibres in the corpus callosum.

In the corpus callosum a deposit of fine Marchi granules was scattered over the splenium in an open and irregular manner, with a small extension anteriorly along the neighbouring inferior border of the body. The splenial deposit was slightly more extensive and pronounced than that observed in the splenium in experiment 4. There was no Marchi deposit in the anterior commissure (Fig. 2,b).

Experiment 3.-Monkey 47A. Operation 24th June, 1938. Killed 13th, July, 1938.

The lesion involved the upper part of the post-central gyrus, including areas 5 , 2, and 1 . It measured $17.5 \mathrm{~mm}$. sagittally and $13 \mathrm{~mm}$. coronally. The upper margin of the lesion was $5 \mathrm{~mm}$. from the superior border of the brain. Anteriorly the lesion just reached the sulcus centralis, while posteriorly it 
extended along the intraparietal sulcus. Neither sulcus was damaged (Fig. $3, a)$.

There was an even and dense deposit of large Marchi granules in the posterior third of the body of the corpus callosum, and a much fainter deposit more anteriorly in approximately the middle third, where, however, the few granules present were irregularly scattered, with a suggestion of concentrations along the upper and lower margins of the body about its centre. There was no Marchi deposit in the anterior commissure (Fig. 3, b).

$a$
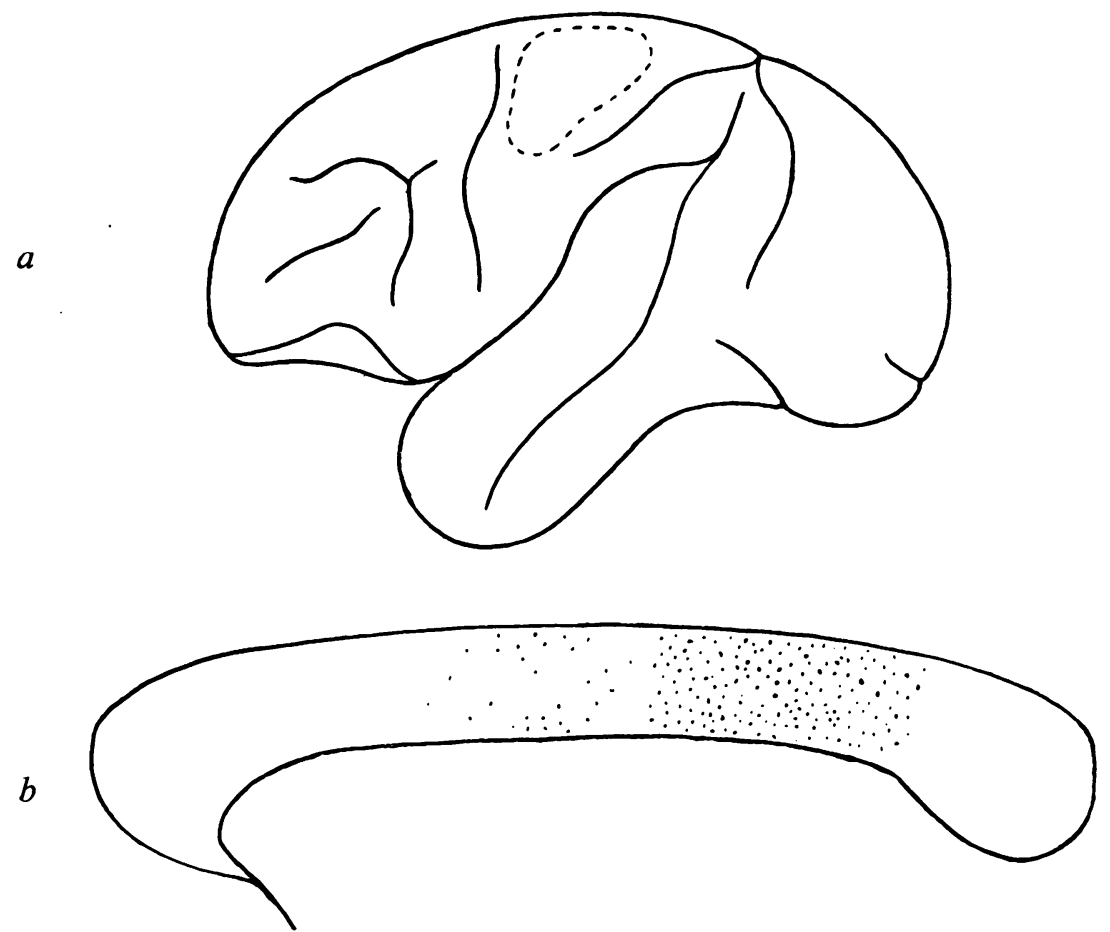

Fig. 3.-The lesion in experiment 3 and the distribution of the degenerated fibres in the corpus callosum.

Experiment 4.-Monkey 49A. Operation 12th May, 1938. Killed 30th May, 1938.

The lesion was an extensive one involving most of the outer surface of the occipital lobe. It measured $28 \mathrm{~mm}$. coronally and $14 \mathrm{~mm}$. antero-posteriorly. The margin of the lesion was $15 \mathrm{~mm}$. from the occipital pole posteriorly and $5 \mathrm{~mm}$. and $3 \mathrm{~mm}$. from the inferior and superior borders of the brain respectively. Anteriorly it followed the sulcus lunatus, but remained separated from it by a distance of $1 \mathrm{~mm}$. The lesion involved area 17 and the immediately adjacent part of area 18 (Fig. 4, a).

Large Marchi granules were observed in the septum pellucidum, at the junction of the posterior third and anterior two-thirds of the body, and a finer deposit in the splenium of the corpus callosum. In the septum the granules 
were clustered anteriorly and posteriorly, and their arrangement suggested that they were located in fibres directed anteroposteriorly across the septum. The granules were openly and irregularly distributed over the tip and lower aspect of the splenium, with an extension of more densely arranged granules reaching forwards for a short distance along the lower margin of the body. The small patch of the body was well separated from the other deposits. There was no Marchi deposit in the anterior commissure (Fig. 4, b).

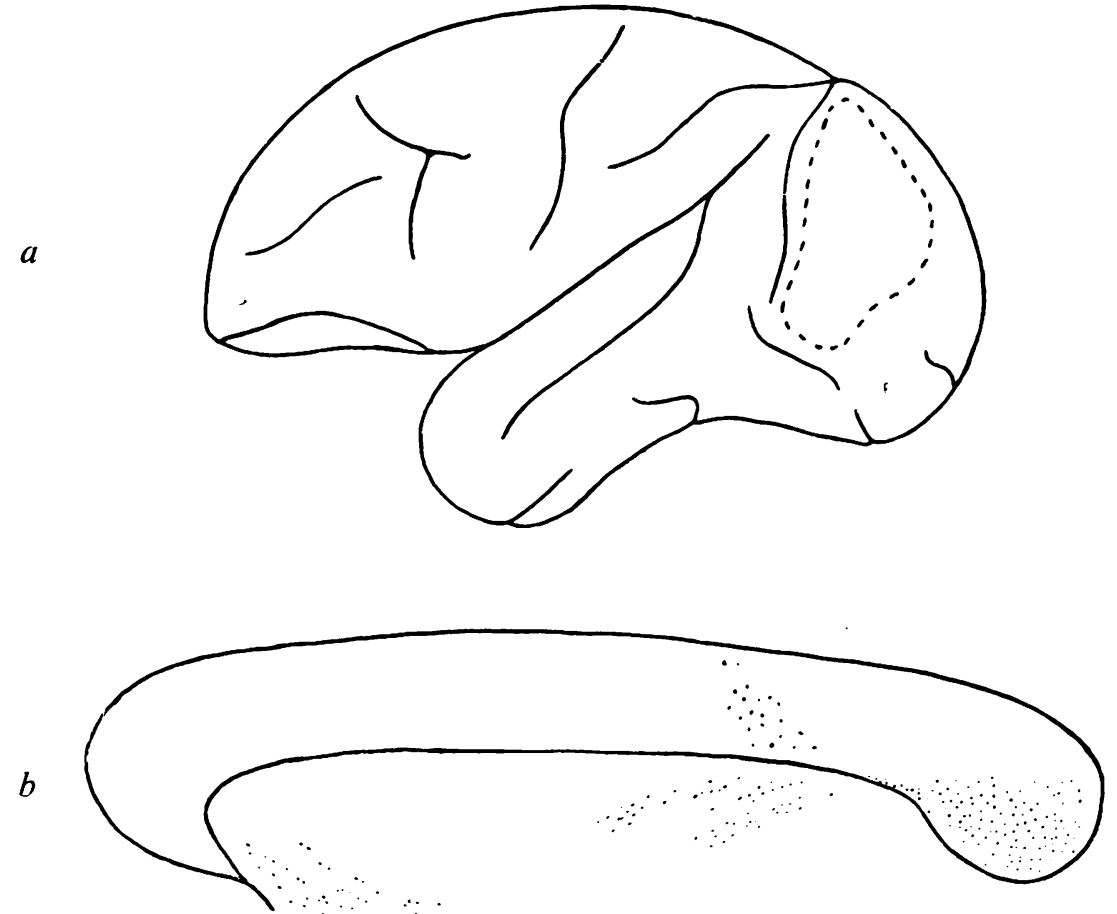

Fig. 4.-The lesion in experiment 4 and the distribution of the degenerated fibres in the corpus callosum.

Experiment 5.-Monkey 50A. Operation 24th May, 1938. Killed 10th June, 1938.

The lesion was located in the prefrontal region above the sulcus arcuatus, the upper extremity of which was involved in the lesion. The latter involved areas 6 and 9 . Its dimensions were $19 \mathrm{~mm}$. antero-posteriorly, with a maximum coronal diameter of $12 \mathrm{~mm}$. Antero-inferiorly the margin of the lesion was in contact with the sulcus rectus, while superiorly a distance of $5 \mathrm{~mm}$. separated it from the superior border of the brain (Fig. 5,a).

There was a heavy deposit of fine Marchi granules distributed evenly over the genu and a little more than the anterior third of the body of the corpus callosum. Posteriorly this deposit thinned away abruptly over the middle third of the body, where, in comparison, the granules were much fewer in number and more openly arranged. The deposit in the anterior third of the 
body was more extensive and the granules more closely packed than in experiment 1. There was no Marchi deposit in the anterior commissure (Fig. 5, b).

Experiment 6.-Monkey 52A. Operation 26th May, 1938. Killed 13th June, 1938.

This lesion measured $16 \mathrm{~mm}$. in length and $10 \mathrm{~mm}$. in width. It was situated in the inferior portion of the superior temporal gyrus, but did not involve the Sylvian fissure or temporal sulcus. It was localized to area 22 (Fig. 6, a).

The main Marchi deposit, in the form of large granules, was spread over the
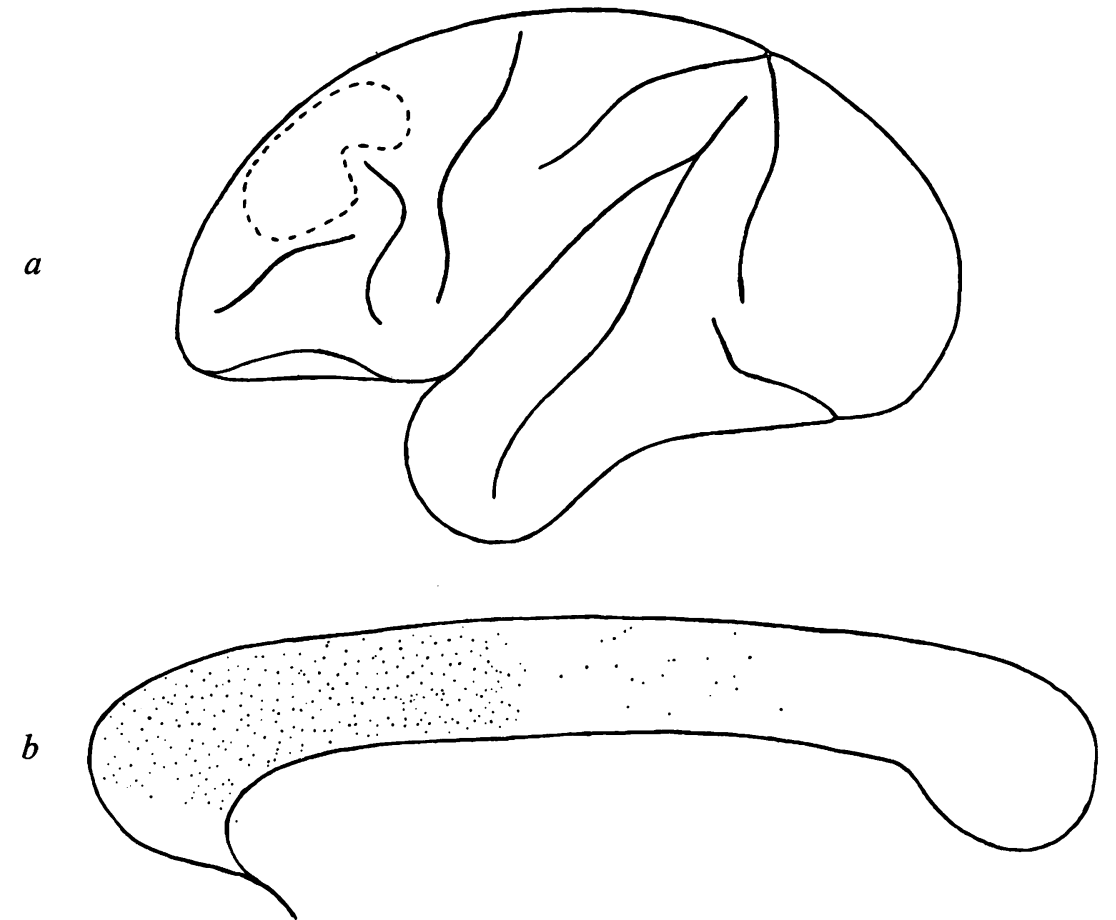

Fig. 5.-The lesion in experiment 5 and the distribution of the degenerated fibres in the corpus callosum.

middle third of the body of the corpus callosum. Isolated granules were also diffusely scattered over the posterior third, where, in addition, there was a linear concentration of granules along the lower margin. In this case the anterior commissure showed a definite deposit in the form of large Marchi granules scattered diffusely over the rostral half of the commissure (Fig. 6, b).

\section{Discussion}

Current statements regarding the distribution of commissural fibres in the corpus callosum mostly have as their basis the observations of Mingazinni (1926), whose studies on the myelination of fibres in the corpus callosum of the human brain showed that frontal fibres cross by way of the genu, that the 
body contains fibres from the first frontal, precentral, parietal, paracentral, and insular territories, while parietal, occipital, lingual, and angular fibres are confined to the splenium. The observations made in this experimental investigation on the monkey's brain are not entirely in accord with Mingazinni's observations on the human brain. While it is probable that the two are not closely comparable, because of the different degree of relative development of some of the cortical areas, it also appears that the experimental method is likely

$a$
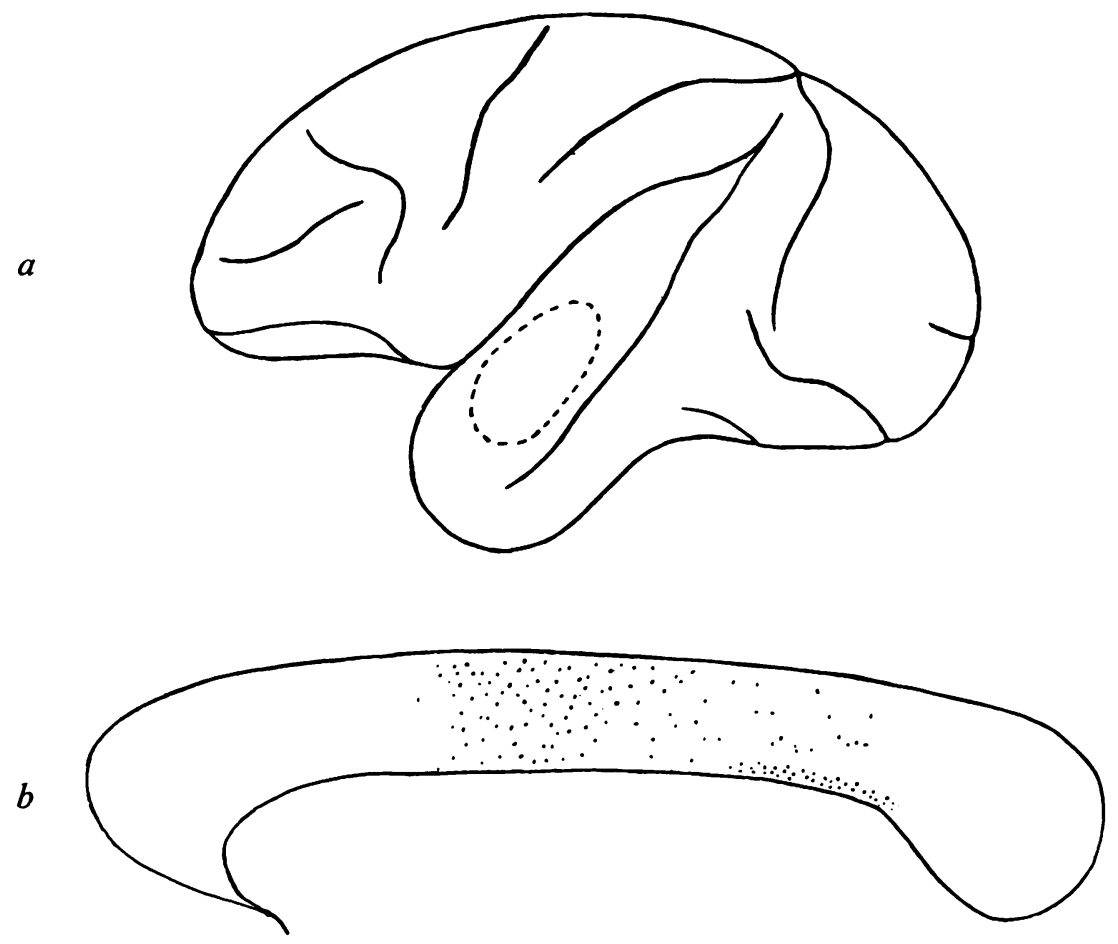

$\because$

Fig. 6.-The lesion in experiment 6 and the distribution of the degenerated fibres in the corpus callosum.

to yield more precise information regarding the location in the corpus callosum of commissural fibres from different cortical areas.

All the cortical lesions which were made gave origin to a degeneration of callosal fibres. Since each lesion involved, except in the case of experiment 6 , a group of cyto-architectural areas, it is impossible to assess with any accuracy the relative contribution from each particular area.

Callosal fibres from the frontal lobe occupy approximately the genu and the anterior third of the body of the corpus callosum, and the nature of the deposit suggests that a proportionately large number of commissural fibres arise in the frontal areas. In experiment 5, the smaller of the two frontal lesions, 
the deposit was denser and occupied a slightly more extensive area than in experiment 1 , and this is perhaps due to the preponderance of commissural fibres coming from the premotor area. The deposit in the splenium was very similar in the two experiments involving the occipital cortex, though in experiment 4 it was not restricted to this portion of the corpus callosum. The amount of the deposit observed in the two cases suggests that more commissural fibres in the splenium arise from the peristriate areas than from the striate area. It is generally believed (Valkenburg, Poliak et al.) that area 17 gives no commissural fibres, and, if this is true, the large number of callosal fibres in experiment 4 must arise in the small strip of area 18 which is presumably included in the lesion. It should be noted, however, that Poliak (1927) has demonstrated that callosal commissural fibres arise from the area striata in the cat. The surface area of the lesion in experiment 2 exceeds that belonging to area 18 in experiment 4, and the inference drawn from this observation is that area 19 is not represented in the corpus callosum to the same extent as area 18 . If the area striata is eliminated, the splenial fibres revealed in this study presumably take origin in areas 18 and 19. It is difficult to comment on the nature of the fibres occupying the posterior third of the body of the corpus callosum and the septum pellucidum. Though they are well separated from the remainder they presumably take origin in area 18 ; perhaps they represent fibres of a heterotopic type which are directed more anteriorly than the others.

In both the temporal and parietal lesions there was a heavy deposit scattered irregularly over the posterior two-thirds of the body of the corpus callosum, but the patches where the granules showed a relative concentration differed in two lesions and suggest that the main mass of fibres from the temporal lobe occupy a position in the corpus callosum anterior, though not inferior, to those coming from the parietal lobe. In the case of the temporal lesion additional commissural fibres were observed to cross by way of the anterior commissure. It seems certain that these fibres come from the temporal cortex, because in this series the lesions were localized to the cortex, and this would exclude any damage to the basal ganglia or subjacent fibre tracts in this case, an explanation which is believed by some to account for the deposit in the anterior commissure following temporal lesions.

Comparing the size of the lesions and the amount of the deposit in each experiment it appears that, so far as the regions studied here are concerned, the parietal lobe gives origin to most commissural fibres and is followed by the frontal, temporal, and occipital territories in that order ; and that, while parietal and temporal fibres are spread over a greater area of the corpus callosum, those coming from the frontal lobe show a greater concentration. An interesting point is the extensive callosal areas over which fibres from relatively small cortical regions are dispersed. In general, the frontal fibres occupy the genu and anterior third of the body and the occipital fibres the splenium. In the middle third, frontal, parietal, and temporal fibres overlap, with the latter predominating, while parietal, temporal, and occipital fibres are distributed over the posterior third in that order of importance.

From the distribution of the deposit it appears that localization in the 
corpus callosum is of a very general type. Not only are the commissural fibres from some cortical areas diffusely spread over the corpus callosum, but there is also an overlap of fibres coming from different areas in the same lobe, and also, apparently, from areas in different lobes (Fig. 7).

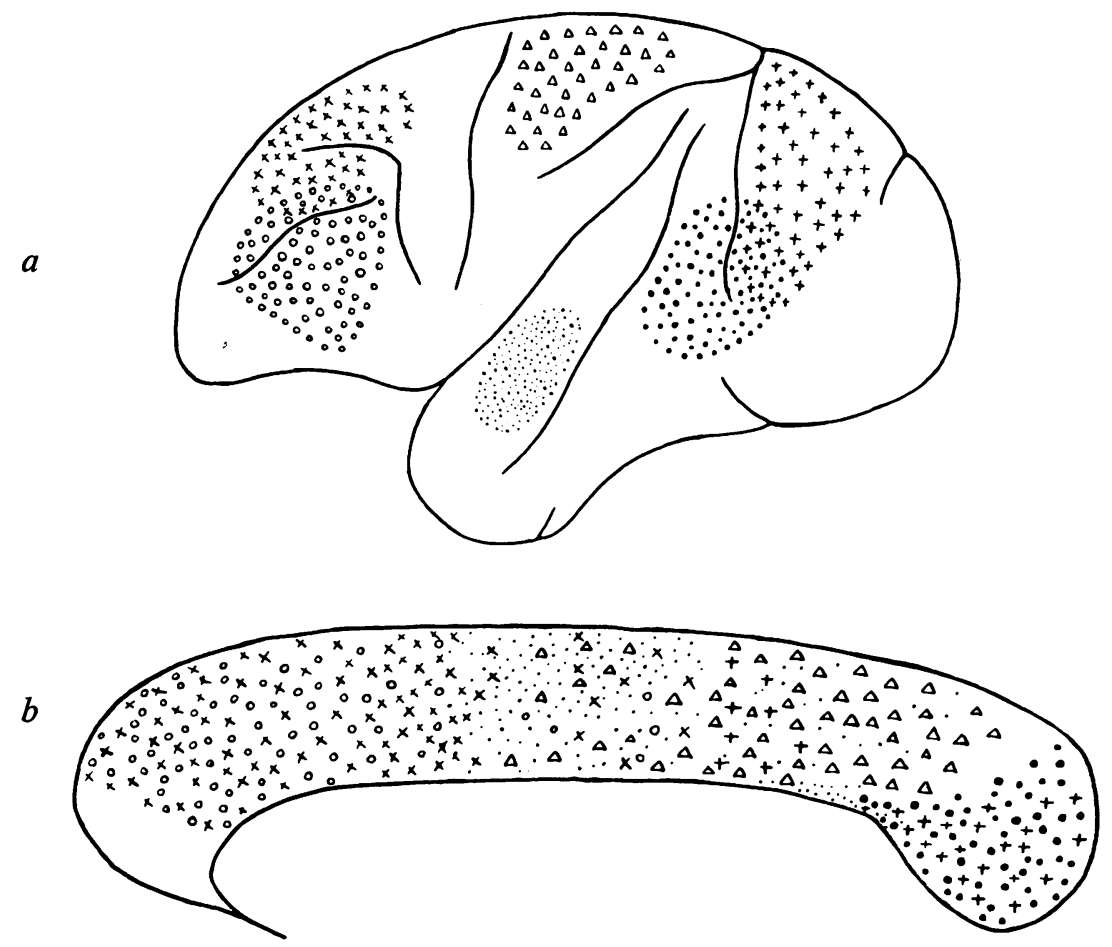

Fig. 7.-Diagram illustrating the degree of overlap in the corpus callosum of fibres coming from different cortical areas.

\section{Summary}

The presence and situation of commissural fibres in the corpus callosum, as shown by a study of degenerating fibres following cortical lesions, are described.

Areas studied in the frontal, parietal, occipital, and temporal lobes were all found to contribute to the callosal system.

The position occupied by the fibres is discussed, with the general conclusion that the frontal fibres are spread over chiefly the genu and anterior third of the body, with some occupying the middle third, the occipital over the splenium and posterior third of the body, and the parietal and temporal over the posterior two-thirds of the body with those from the temporal occupying a position anterior to those coming from the parietal. The existence of commissural fibres from the temporal lobe crossing by way of the anterior commissure is confirmed. The amount of the Marchi deposit recorded suggests that, from areas of equal extent, the parietal cortex contributes relatively the greatest 
number of commissural fibres, and then the cortex of the frontal, temporal, and occipital lobes in that order.

It is a pleasure to acknowledge the advice and assistance which Professor Le Gros Clark has so kindly placed at my disposal throughout the investigation.

\section{REFERENCES}

Beevor, C. E. (1892). Philos. Trans., 182, 135.

Hamilton, D. J. (1885). J. Anat. Physiol., 19, 385. (1885). Brain, 8, 145.

Ironside, L., and Guttmacha, M. (1929). Ibid., 52, 442.

Kappers, C. U. Ariens, Huber, G. C., and Crosby, E. C. (1936). The Comparative Anatomy of the Nervous System of Vertebrates, including Man. Macmillan, New York.

Mellus, E. L. (1904). Johns Hopk. Hosp. Bull., 12, 108.

Mingazinni, G. (1926). Dtsch. Z. Nervenheilk., 94, 168.

Muratow, W. (1893). Neurol. Zbl., 12, 316.

Poliak, S. (1927). J. comp. Neurol., 44, 197.

- (1932). The Main Afferent Fibre Systems of the Cerebral Cortex in Primates. Univ. Calif. Pub. in Anat., 2,

Sherrington, C. S. (1889). J. Physiol., 10, 429.

Sunderland, S. (1938). J. Anat., Lond., 73, 120.

van Valkenburg, C. T. (1913). Brain, 36, 119.

Villaverde, J. de (1919). Schweiz. Arch. Neurol. Psychiat., 4, 45. 